\title{
Morbi-mortalidade em cirurgia de correção de aneurisma de aorta em um hospital público de Fortaleza-CE: estudo retrospectivo
}

\author{
Ana Lígia Coelho Ribeiro', Érica da Rocha Pereira², Maria do Socorro Quintino Farias ${ }^{3}$, \\ Suzy Maria Montenegro Pontes ${ }^{2}$, Thiago Brasileiro de Vasconcelos ${ }^{5}$, Vasco Pinheiro Diógenes Bastos ${ }^{6}$
}

\begin{abstract}
RESUMO
Objetivo: Identificar as complicações pós-operatórias cardíacas e não-cardíacas e fatores de risco para doença cardiovascular nas cirurgias de correção de aneurisma de aorta. Metodologia: Estudo documental, descritivo e retrospectivo com análise quantitativa dos dados. A coleta de dados realizou-se em um hospital publico de referência no tratamento de doenças cardíacas na cidade de Fortaleza/CE. A amostra foi composta por 23 prontuários dos anos 2007 a 2009, e foram exclusos os prontuários que não continham as informações completas. Resultados: O IMC esteve dentro dos limites normais. $O$ tabagismo esteve entre $43 \%$ dos homens e $71,4 \%$ das mulheres. A hipertensão em $93,75 \%$ dos homens e $57,1 \%$ nas mulheres. As complicações cardíacas foram $35,7 \%$ e $64,2 \%$ não cardíacas. As complicações mais incidentes dentre as não cardíacas são a hipotermia $(21,73 \%)$ e relacionadas à incisão cirúrgica (21,73\%). Conclusão: As complicações pósoperatórias que se destacaram foram as não cardíacas, principalmente a hipotermia e as relacionadas à incisão cirúrgica.
\end{abstract}

Descritores: Aneurisma de Aorta; Morbimortalidade; Complicações Pós-operatórias.

\section{Morbi-mortality in surgery of the aorta aneurysm correction in a Public Hospital of Fortaleza-CE: a retrospective study}

\begin{abstract}
Objective: This study aims to identify the post-operative complications of cardiac and non-cardiac and risk factors for cardiovascular disease in surgeries-correction aneurysm of aorta. Methodology: This study was descriptive and retrospective documentary, with quantitative analysis of the data. Data collection took place in the Hospital reference in city of Fortaleza/CE. The sample was composed of the medical records of patients who had surgery to fix aneurysm of aorta in that Hospital between 2007 and 2009, and from the patient that did not contain the information necessary to complete this study data were recorded in own form prepared by the researcher. Results: BMI was within normal limits. Cigarette smoking was $43 \%$ among men and $71.4 \%$ women. Hypertension in $93.75 \%$ of men and $57.1 \%$ in women. The cardiac complications were $35.7 \%$ and $64.2 \%$ non-cardiac. The most incidents complications among noncardiac are hypothermia $(21.73 \%)$ and related to the surgical incision (21.73\%). Conclusion: Postoperative complications that stood out were the noncardiac mainly hypothermia and related to surgical incision.
\end{abstract}

Descriptors: Aneurysm of Aorta; Morbi-mortality; Post-operative Complications.

\footnotetext{
${ }^{1}$ Fisioterapeuta graduada pelo Centro Universitário Estácio do Ceará (FIC), Fortaleza, CE, Brasil.

${ }^{2}$ Especialista em Fisioterapia Respiratória e Cardiovascular pela Universidade de Fortaleza (UNIFOR), Fortaleza, CE, Brasil.

${ }^{3}$ Mestre em Ciências Fisiológicas pela Universidade Estadual do Ceará (UECE), Fortaleza, CE, Brasil.

${ }^{5}$ Mestre em Farmacologia pela Universidade Federal do Ceará (UFC), Fortaleza, CE, Brasil.

${ }^{6}$ Doutor em Farmacologia pela Universidade Federal do Ceará (UFC), Fortaleza, CE, Brasil.
} 


\section{Introdução}

Aneurisma da aorta é uma dilatação localizada, maior que $50 \%$ do diâmetro da luz presumida do vaso em um determinado segmento aórtico ${ }^{1}$. Classifica-se em verdadeiro, quando a dilatação estiver presente em todos os segmentos constituintes da parede do vaso; falso, quando há presença de um trombo substituindo uma das camadas da parede do vaso; e dissecção aguda da aorta, quando a formação aneurismática é decorrente de uma dissecção ocorrida inicialmente ${ }^{2}$.

Crawford em 1986 dividiu os Aneurismas de Aorta torácica em tipo I, com início após a artéria subclávia esquerda até abaixo dos ramos viscerais; tipo II, com início após a artéria subclávia esquerda até a bifurcação da aorta; tipo III, entre a $6^{\circ}$ costela e as artérias renais e o tipo IV, com início abaixo do diafragma até as artérias renais ${ }^{3}$.

Novero ${ }^{4}$ descreve a etiologia dos aneurismas como de origem degenerativa em aproximadamente $80 \%$ dos pacientes, com risco de ruptura e morte em um ano de acompanhamento. A dissecção aórtica é referida em estudos como a afecção mais temida e duas vezes mais frequente do que a ruptura da aorta, sendo esta um dos fatores de risco para a ruptura de um aneurisma, tal como o diâmetro maior que $5 \mathrm{~cm}$, evolução no crescimento do aneurisma, tabagismo, doença pulmonar obstrutiva crônica, hipertensão arterial sistêmica, insuficiência renal crônica e doenças degenerativas da aorta ${ }^{5,6}$.

A correção cirúrgica do aneurisma é realizada quando seu diâmetro atinge ou ultrapassa $5,5 \mathrm{~cm}$ para homens e 5,0 $\mathrm{cm}$ para mulheres ou quando ocorre expansão do diâmetro transverso $\geq 5 \mathrm{~cm}$ em um ano. Além disso, a indicação de cirurgia deve ser norteada por considerações que levem em conta o risco de ruptura do aneurisma, confrontando-o com o risco cirúrgico e a expectativa de vida do paciente ${ }^{7,8}$.

Devido a alta taxa de morbi-mortalidade da cirurgia convencional, Parodi e Palmaz em 1991 relataram o uso de uma nova técnica cirúrgica para a correção dos aneurisma de aorta abdominal ${ }^{9}$. Desde essa época, a técnica endovascular esta sendo utilizada visando a redução da morbi-mortalidade peri e pós-operatória a curto e longo prazo desses pacientes submetidos a correção cirúrgica ${ }^{9,10}$. Novero ${ }^{11}$ acredita que com a experiência acumulada e o desenvolvimento de endopróteses mais seguras e flexíveis, a técnica endovascular em pacientes de alto risco entra como uma nova e eficaz alternativa de correção de aneurisma de aorta abdominal.

As doenças cardiovasculares são as principais causas de internação em setor público em todo o Brasil, constituindo assim um problema de saúde pública. Os achados científicos sobre o perfil desses pacientes, e a morbi-mortalidade em relação à correção cirúrgica de aneurisma de aorta, ainda são escassas, portanto, este estudo objetiva identificar as complicações pós-operatórias cardíacas e não-cardíacas e fatores de risco para doença cardiovascular nas cirurgias de correção de aneurisma de aorta.

\section{Metodologia}

O presente estudo foi documental, descritivo e retrospectivo com análise quantitativa dos dados. A coleta de dados realizou-se no período de junho a agosto de 2010 em um hospital de referência no tratamento de doenças cardiovasculares, localizado na cidade de Fortaleza/CE, após aprovação do Comitê de Ética e Pesquisa do Hospital de Messejana Dr. Calos Sturdart Gomes (CEP/HM n 10239073-8).

A amostra foi composta pelos prontuários dos pacientes que realizaram cirurgia de correção de aneurisma de aorta no referido Hospital, sendo inclusos na pesquisa, os prontuários dos pacientes que realizaram a cirurgia de correção de aneurisma de aorta em qualquer dos seus segmentos no período de Janeiro de 2007 à Dezembro de 2009. Foram exclusos da coleta de dados os prontuários que não continham as informações completas necessárias a este estudo. Realizou-se uma busca ativa dos registros das cirurgias para correção de aneurisma de aorta, identificados inicialmente no banco de dados do referido hospital. Estes foram registrados em formulário próprio elaborado pelo autor, contendo dados de identificação, peso, altura, complicações cardíacas e não cardíacas.

Para o cálculo do Índice de Massa Corpórea (IMC) foi considerado magreza (IMC $<18,5 \mathrm{~kg} / \mathrm{m}^{2}$ ), ideal (entre $18,5 \mathrm{e}$ $24,9 \mathrm{~kg} / \mathrm{m}^{2}$ ), sobrepeso (entre 25 a $29,9 \mathrm{~kg} / \mathrm{m}^{2}$ ), obesidade 1 (entre 30 e $34,9 \mathrm{~kg} / \mathrm{m}^{2}$ ), obesidade 2 (entre 35 a $39,9 \mathrm{~kg} / \mathrm{m}^{2}$ ) e obesidade mórbida (maior ou igual a $40 \mathrm{~kg} / \mathrm{m}^{2}$ ). Este estudo obedeceu as normas da Resolução 196/96 do Conselho Nacional de Saúde. Durante e após a coleta de dados, a pesquisadora manteve o sigilo das informações. 


\section{Resultados}

No período pesquisado, submeteram-se a correção de aneurisma de aorta 23 pacientes, sendo $69,44 \%(n=16)$ do sexo masculino e $30,43 \%(n=7)$ do sexo feminino.

O Índice de Massa Corpórea (IMC) teve média de $24,05 \mathrm{~kg} / \mathrm{m}^{2}$ para homens e $22,61 \mathrm{~kg} / \mathrm{m}^{2}$ para as mulheres, estando ambos os sexos com o IMC dentro da normalidade $\left(18,5\right.$ a $\left.24,99 \mathrm{~kg} / \mathrm{m}^{2}\right)$.

Na tabela 1 observa-se que o fator de risco mais predominante nos homens é a hipertensão $(93,75 \% ; n=15)$ e nas mulheres é o tabagismo $(71,4 \% ; n=5)$.

Tabela 1 - Distribuição dos dados de acordo com os fatores de risco para Doença Arterial Coronariana (DAC) em pacientes que realizaram correção cirúrgica de aneurisma de aorta, Fortaleza/CE.

Fonte: Dados da pesquisa.

\begin{tabular}{c|c|c|c|c}
\hline \multirow{2}{*}{ VARIÁVEL } & \multicolumn{2}{|c|}{ Homem } & \multicolumn{2}{c}{ Mulher } \\
\cline { 2 - 5 } & $\mathrm{n}$ & $\mathrm{f}$ & $\mathrm{n}$ & $\mathrm{f}$ \\
\hline Tabagismo & 7 & $43 \%$ & 5 & $71,4 \%$ \\
\hline Hipertensão & 15 & $93,75 \%$ & 4 & $57,1 \%$ \\
\hline Etilismo & 4 & $25 \%$ & 2 & $28,5 \%$ \\
\hline Diabetes & 4 & $25 \%$ & 0 & - \\
\hline
\end{tabular}

A tabela 2 expõe as complicações pós-operatórias de correção cirúrgica de aneurisma de aorta mais comuns no hospital pesquisado. As complicações $(n=14)$ foram $64,2 \%(n=9)$ não-cardíacas e $35,71 \%(n=5)$ cardíacas. As complicações mais incidentes dentre as não-cardíacas são a hipotermia $(21,73 \%)$ e relacionadas à incisão cirúrgica $(21,73 \%)$.

Tabela 2 - Complicações Pós-operatória registradas em pacientes que realizaram correção cirúrgica de aneurisma de aorta, Fortaleza/CE.

\begin{tabular}{c|c}
\hline \multicolumn{2}{c}{ • Complicações Cardíacas } \\
\hline Hipotensão & $\mathrm{n}=2(8,6 \%)$ \\
\hline Derrame Pericárdico & $\mathrm{n}=1(4,3 \%)$ \\
\hline Disfunção do VE & $\mathrm{n}=1(4,3 \%)$ \\
\hline Taquicardia & $\mathrm{n}=2(8,6 \%)$ \\
\hline Hipertensão & $\mathrm{n}=5(21,73 \%)$ \\
\hline \multicolumn{2}{c}{ • Complicações Não cardíacas } \\
\hline Hipotermia & $\mathrm{n}=5(21,73 \%)$ \\
\hline Relacionadas ao dreno & $\mathrm{n}=3(13,04 \%)$ \\
\hline Relacionadas à incisão cirúrgica & $\mathrm{n}=5(21,73 \%)$ \\
\hline Broncoespasmo & $\mathrm{n}=1(4,3 \%)$ \\
\hline Atelectasias & $\mathrm{n}=1(4,3 \%)$ \\
\hline Gastrointestinais & $\mathrm{n}=1(4,3 \%)$ \\
\hline Infecção & $\mathrm{n}=1(4,3 \%)$ \\
\hline
\end{tabular}

Fonte: Dados da pesquisa.

Durante a análise dos dados foi observado que um paciente teve tempo de internação pós-cirúrgico aumentado (18 dias) devido a episódios frequentes de vômitos.

Foi avaliado no presente estudo que o tempo médio de internação pós-cirúrgico foi de 15,3 dias. Não houve óbitos no período pesquisado.

Estudos posteriores podem ser realizados com intuito de identificar métodos de diagnósticos mais apropriados para detectar risco de desenvolvimento de aneurisma de aorta, o que poderia diminuir os riscos cirúrgicos e o tempo de internação hospitalar. 


\section{Discussão}

A cirurgia endovascular para a correção do aneurisma da aorta abdominal não está livre de complicações, que podem ocorrer durante ou após o procedimento. Contudo, a escolha desta modalidade terapêutica tem sido justificada pela comparação à cirurgia convencional ${ }^{12}$.

Bonamigo, Lucas, Junior ${ }^{13}$ e Metzer et al. ${ }^{14}$ estudaram que a prevalência dos aneurismas de aorta pode ser até seis vezes maior nos homens, um percentual próximo ao encontrado no presente estudo.

Segundo Koning et al. ${ }^{15} 0$ aumento da morbimortalidade dos pacientes que realizaram cirurgia para correção endovascular de aneurisma de aorta não está definida, não sabendo ainda se está ou não relacionada à técnica cirúrgica. Bonamigo, Lucas, Pereira ${ }^{16}$ estudou os riscos da correção cirúrgica de aneurisma de aorta em pacientes jovens $(<50$ anos) onde encontrou que a mortalidade perioperatória é nula e a sobrevida é boa.

Entretanto, Batt et al. ${ }^{17}$ classifica as complicações pós-operatórias em precoces e tardias. As imediatas mais comuns estão ligadas ao procedimento cirúrgico como sangramentos persistentes com consumo de fatores da coagulação, lesões vasculares, lesão de vísceras parenquimatosas, lesão dos ureteres, embolias, hipotermia e tromboses. As complicações tardias são pseudo-aneurismas anastomóticos, fístulas aorto-entéricas, trombose e infecção do enxerto. Os pacientes com antecedente de tabagismo e doença pulmonar obstrutiva crônica apresentam fator de risco para desenvolvimento de insuficiência respiratória ${ }^{17,18}$.

Estudo realizado com o intuito de relacionar o IMC com o risco de desenvolver hipertensão arterial constatou que aproximadamente metade dos homens e mais de um terço das mulheres apresentaram IMC acima do limite superior dos valores considerados normais $\left(\geq 25 \mathrm{~kg} / \mathrm{m}^{2}\right)^{19}$ indo de encontro aos resultados apresentados no presente estudo.

Segundo Carvalho et al. ${ }^{20} \mathrm{e}$ Silva et al. ${ }^{21}$ o paciente ser tabagista e/ou hipertensivo aumenta o risco de desenvolver aneurisma de aorta. Em estudo realizado na Universidade Federal de São Paulo, 15,5\% dos pacientes estudados eram etilistas, $48,5 \%$ hipertensos e $20,5 \%$ tabagistas. A sobrevida desses pacientes foi considerada boa pelos autores do estudo $^{22}$, concordando com nossos dados, pois obervamos alta taxa de fatores de risco para o desenvolvimento de doenças artérias, porém após a cirurgia não houve óbitos no período pesquisado.

Becker, Bonamigo, Faccini ${ }^{23}$ e Valle et al. ${ }^{24}$ quando avaliaram a influencia dos fatores de risco para a mortalidade em pós-operatório de correção cirúrgica de aneurisma de aorta abdominal, observaram que não houve aumento estatisticamente significativo da mortalidade em pacientes com insuficiência renal, diabetes mellitus, hipertensão arterial sistêmica, acidente vascular cerebral e infarto agudo do miocárdio.

As intercorrências de ordem cardíacas são extremamente comuns no pós-operatório de aneurismas da aorta, sendo que alguns autores relatam que $40 \%$ desses pacientes são coronariopatas e que até $10 \%$ dos mesmos devem receber procedimento cardíaco prévio ao tratamento cirúrgico do aneurisma da aorta. A mortalidade de pacientes com diagnóstico de doença coronariana grave (lesão de tronco coronariano, três vasos e angina instável) triplica em relação aos pacientes sem tal achado ${ }^{25}$.

Quando se estudou a relação entre coronariopatias em idosos e o desenvolvimento de aneurisma de aorta verificou que a utilização do Doppler ultrassom para o diagnostico precoce das dilatações da aorta abdominal diminui a mortalidade por ruptura ${ }^{26}$.

No estudo de Carvalho et al. ${ }^{27}$ prevaleceram as complicações cardíacas, o que difere do resultado observado no presente estudo, onde se prevalecem as complicações não-cardíacas. As complicações tardias mais comuns estão relacionadas à incisão cirúrgica ${ }^{17,18}$.

No presente estudo dentre as complicações cardíacas, a mais comum foi a hipertensão (21,73\%), seguida de taquicardia (8,6\%). Kusomoto ${ }^{28}$ estudou que os pacientes tabagistas, diabéticos, hipertensivos são mais propensos a desenvolver doenças nas artérias coronarianas. Menezes, Luccas, Matsui ${ }^{29}$ constatou que a prevalência de Doença Arterial Coronariana em pacientes com aneurisma de aorta foi elevada.

Segundo Castro Júnior, Wietzcoski, Mespaque ${ }^{25}$ a causa mais frequente de aneurisma de aorta é a aterosclerose, principalmente nos de localização abdominal onde se encontram $75 \%$ dos aneurismas ateroscleróticos.

Complicações gastrointestinais após cirurgias da aorta acontecem em 6,6 a 21\% dos casos e estão associadas à grande morbidade e aumento da mortalidade ${ }^{30}$.

Apesar da alta ocorrência da hipotermia no pós-operatório da cirurgia de correção de aneurisma de aorta, não foi encontrado relatos na literatura que registrem tal acontecimento. 


\section{Considerações Finais}

Por meio desse estudo observou-se que a ocorrência de aneurisma de aorta ainda é considerada alta, e mais incidente no sexo masculino.

Verificou-se que a presença de hipertensão, tabagismo, etilismo e diabetes são predeterminantes para o desenvolvimento de aneurisma de aorta. $\mathrm{O}$ índice de pacientes hipertensivos e tabagistas foram significantes. O IMC esteve dentro dos limites considerados normais.

As complicações pós-operatórias que se destacaram foram as não cardíacas, principalmente a hipotermia e as relacionadas à incisão cirúrgica.

Apesar de muitos dos estudos avaliados a respeito da relação entre DAC e aneurisma da aorta destacarem a relação direta dessas duas patologias, no presente estudo foram registrados pacientes com DAC prévia. Porém, a taxa de pacientes com hipertensão e tabagismo foi alta. Isso certamente contribui para um prognóstico não satisfatório.

\section{Referências Bibliográficas}

1. Johnston KW, Rutherford RB, Tilson MD, Shah DM, Hollier L, Stanley JC. Suggested standards for reporting on arterial aneurysms. J. Vasc Surg. 1991; 13(3):452-8.

2. Porto CC. Doenças do Coração: prevenção e tratamento. 2. ed. São Paulo: Guanabara Koogan; 2005.

3. Crawford ES, Crawford JL, Safi JH, Coselli JS, Hess KR, Brooks B et al. Thoracoabdominal aortic aneurysms: preoperative and intraoperative factors determining immediate and long-term results of operations on 605 patients. J Vasc Surg. 1986; 3(3):389-404.

4. Novero ER, Metzger PB, Obregon J, Marco VLA, Rossi FH, Moreira SM et al. Tratamento endovascular das doenças da aorta torácica: análise dos resultados de um centro. Radiol Bras. 2012; 45(5):251-258.

5. Koning GG, Hobo R, Laheij RJF, Buth J, Van Der Vliet JA. Medicações referentes às complicações após correção de aneurisma da aorta abdominal endovascular. Rev Bras Cir Cardiovasc, 2006; 21(2):149-154.

6. Davies RR, Goldstein LJ, Coady MA, Tittle SL, Rizzo JA, Kopf GS et al. Yearly rupture or dissection rates for thoracic aortic aneurysms: simple prediction based on size. Ann Thorac Surg. 2002; 73(1):17-27.

7. Silvestre JMS, Motta F, Sardinha WE, Filho DM, Thomazinho F, Silvestre GS et al. Tratamento endovascular do aneurisma da aorta abdominal infrarrenal em pacientes com anatomia favorável para o procedimento - experiência inicial em um serviço universitário. J Vasc Bras. 2011; 10(1):31-39.

8. Pontes JCDV, Duarte JJ, Silva AD, Dias AMSAS. Correção endovascular de aneurisma de aorta abdominal em complicação tardia de dissecção de aorta tipo A. Rer Bras Cir Cardiovasc. 2012; 27(4):645-647.

9. Parodi JC, Palmaz IC, Barone HD. Transfemural intraluminal graft implantation for abdominal aortic aneurysms. Ann Vasc Surg. 1991; 5(6):491-9.

10. Oliveira FAC, Campedelli FL, Amorelli CES, Filho JEC, Gibbon DR, Barreto JC et al. Tratamento endovascular da oclusão de ramo ilíaco de endoprótese bifurcada de aorta abdominal: trombectomia rotativa e aspirativa seguida de angioplastia com stent primário. J Vasc Bras. 2012; 11(3):212-218.

11. Novero ER, Metzger PB, Angelieri FMR, Colli MBO, Moreira SM, Izukawa NM et al. Correção endovascular do aneurisma da aorta abdominal: análise dos resultados de um único centro. Radiol Bras. 2012; 45(1):1-6.

12. Bastos RM, Filho AR, Blasbalg R, Caffaro RA, Karakhanian WK, Esteves FP et al. Trombose na endoprótese do aneurisma da aorta: avaliação por TC multidetector. Rer Assoc Med Bras. 2011; 57(1):31-34.

13. Bonamigo TP, Lucas ML, Junior NE. Tratamento cirúrgico dos aneurismas da aorta abdominal: existe diferença dos resultados entre homens e mulheres? J. vasc. bras. 2006; 5(2):101-108.

14. Metzger PB, Novero ER, Rossi FH, Moreira SM, Barbato HA, Izukawa NM et al. Tratamento Endovascular dos Aneurismas da Aorta Abdominal com Anatomia Complexa: Resultados Preliminares com a Segunda Geração de Endoprótese com Arcabouço Metálico Circular. 2012; 20(1):69-76.

15. Koning GG, Vallabhneni SR, Marrewijk CJV, Leurs LJ, Laheij RJF, Buth J. Mortalidade relacionada ao tratamento endovascular do aneurisma da aorta abdominal com o uso dos modelos revisados. Rev Bras Cir Cardiovasc. 2007; 22(1):7-14.

16. Bonamigo TP, Lucas ML, Pereira LC. Resultados da cirurgia do aneurisma da aorta abdominal em pacientes jovens. J. vasc. bras. 2009; 8(2):139-142. 
17. Batt M, Staccini P, Pittaluga P, Ferrari E, Hassen-Khodja R, Declemy S. Late survival after abdominal aortic aneurysm repair. Eur J Vasc Endovasc Surg. 1999; 17(4):338-42.

18. Aun R, Neto FTS, Lederman A, Waksman H. Tratamento endoluminal de aneurismas anastomóticos na aorta abdominal: relato de dois casos. J Vasc Bras. 2006; 5(1):371-81.

19. Sarno F, Monteiro CA. Importância relativa do Índice de Massa Corporal e da circunferência abdominal na predição da hipertensão arterial. Rev Saúde Publica. 2007; 41(5):788-796.

20. Carvalho ATY, Santos AJ, Gomes CAP, Martins ML, Santos VP, Rubeiz RP et al. Aneurisma da aorta abdominal infrarrenal: importância do rastreamento em hospitais do Sistema Único de Saúde na região metropolitana de Salvador - Bahia. J Vasc Bras. 2012; 11(4):289-300.

21. Silva JMF, Alves ASC, Pereira AJM, Pereira EJA, Pedro LM. A dilatação da aorta ascendente é marcador de dilatação da aorta abdominal? Relações entre o diâmetro da aorta torácica avaliada por ecocardiografia e a aorta abdominal estudada por ultrassonografia. Angiol Cir Vasc. 2013; 9(2):52-58.

22. Silva VF, Real DSS, Branco JNR, Catani R, Kim HC, Buffolo E et al. Operação de Bentall e De Bono para correção das doenças da raiz aórtica: análise de resultados a longo prazo. Rev Bras Cir Cardiovasc. 2008; 23(2):256-261

23. Becker M, Bonamigo TP, Faccini FP. Avaliação da mortalidade cirúrgica em aneurismas infra-renais da aorta abdominal. J Vasc Bras . 2002; 1(1):15-21.

24. Valle FH, Costa AR, Pereira EMC, Santos EZ, Junior FP, Bender LP et al. Morbimortalidade em Pacientes acima de 75 Anos Submetidos à Cirurgia por Estenose Valvar Aórtica. Arq Bras Cardiol. 2010; 94(6):720-725.

25. Castro Júnior MAM, Wietzcoski CR, Mespaque CB. Hemoptise como manifestação de aneurisma de aorta torácica descendente. Rev Col Bras Cir. 2004; 31(4):276-277.

26. Heather BP, Poskitt KR, Earnshaw JJ, Whyman M, Shaw E. Population screening reduces mortality rate aortic aneurysm in men. Br J Surg. 2000; 87(6):750-3.

27. Carvalho FC, Brito VPMR, Tribulatto EC, Bellen BV. Estudo prospectivo da morbi-mortalidade precoce e tardia da cirurgia do aneurisma da aorta abdominal. Arq Bras Card. 2005; 84(4):292-296.

28. Kusumoto FM. Fisiopatologia Cardiovascular. 1. ed. São Paulo: Atheneu Editora; 2001.

29. Menezes FH, Luccas GC, Matsui IA. Sobrevida tardia de pacientes submetidos à correção aberta eletiva de aneurisma de aorta abdominal. J. vasc. bras. 2007; 6(3):218-224.

30. Oliveira GJAM, Petnys A, Rabboni E, Neves JMA. Petterle PH, Melo RC et al. Oclusão duodenal após cirurgia da aorta abdominal: relato de caso. J. vasc. bras. 2008; 7(4): 384-388.

\section{Ana Lígia Coelho Ribeiro}

Endereço para correspondência - Rua Eliseu Uchoa Becco, № 600, Bairro: Água Fria, CEP - 60210-270,

Cidade - Fortaleza, CE, Brasil.

E-mail: analigia_2005@hotmail.com

Lattes: http://lattes.cnpq.br/7560595003587994

Érica da Rocha Pereira - ericarocha.rp@hotmail.com

Maria do Socorro Quintino Farias - qfarias@edu.estacio.br

Suzy Maria Montenegro Pontes - suzy.pontes@yahoo.com.br

Thiago Brasileiro de Vasconcelos - thiagobvasconcelos@hotmail.com

Vasco Pinheiro Diógenes Bastos - vascodiogenes@yahoo.com.br

\section{Enviado em 19 de março de 2014.} Aceito em 30 de março de 2015. 